\title{
La tendance des miels à cristalliser : nouvel essai d'approche statistique
}

\author{
T Tabouret 1, JL Berdagué 2, J Lhéritier 3 \\ ${ }^{1}$ UUT, BP 510, 21014 Dijon Cedex; \\ 2 SRV INRA Theix, 63122 Ceyrat; \\ ${ }^{3}$ France-Miel, $B P n^{\circ}$ 5, 39330 Mouchard, France
}

(Reçu le 30 mars 1992; accepté le 15 mai 1992)

\begin{abstract}
Résumé - Cette étude s'efforce de relier la tendance à cristalliser des miels liquides du commerce aux données technologiques de production mesurables sinon maîtrisables. Pour cela, 25 miels typiques ont été pasteurisés puis stockés à $5,10,15$ et $20^{\circ} \mathrm{C}$ et notés, à 8 âges différents, de 0 à 9 selon leur degré de cristallisation. La variance de la note est analysée en fonction de la température et de l'âge. Des régressions linéaires multiples progressives de la note sont faites sur la composition, en glucose fructose et eau, et sur la viscosité initiales. Seul le stockage à $5{ }^{\circ} \mathrm{C}$ se différencie des autres par une période de latence, attribuée à la viscosité initiale élevée, mais ensuite la vitesse de cristallisation est la même. Une explication est proposée en termes de technologie de cristallisation. La note au début du stockage est imprévisible. Elle est grossièrement prévisible à long terme connaissant la composition et la viscosité. Sa variabilité est expliquée à $80 \%$ pour la majorité des miels, mais quelques variétés ont un comportement atypique ce qui limite à $50 \%$ le taux pour l'ensemble des miels étudiés.
\end{abstract}

miel / cristallisation / stockage / étude statistique

\section{INTRODUCTION}

Cette étude prolonge celle de 1987 (Tabouret et al, 1987). Les miels liquides du commerce manquent de stabilité, même lorsqu'ils ont été pasteurisés : leur tendance à cristalliser pendant leur stockage en pots est très préjudiciable à leur qualité. Cette tendance est loin d'être élucidée. Nous nous sommes efforcés de la relier statistiquement aux principales données technologiques de leur élaboration mesurables et plus ou moins maîtrisables.
Les miels étudiés ont été choisis à dessein aussi typés et aussi diversifiés que possible, car l'étude de 1987 avait montré qu'un échantillonnage, au hasard, de la production en sortie d'atelier, ne convenait guère, faute d'une cristallisation suffisante.

Nous avons mesuré les valeurs initiales de leur viscosité et de leur composition en glucose, fructose et eau, car ces variables très importantes résultent du mélange des miels naturels en début de production, et peuvent de ce fait être modulées quelque peu, dans les limites de la 
réglementation selon la dénomination commerciale du produit vendu. Après ce mélange, la composition du miel n'évolue que très peu à la pasteurisation et au stockage, même lors d'un stockage de longue durée (Gonnet, 1965). Nous les avons appelées ci-après «mesures instrumentales", et nous avons apprécié leurs relations avec la «mesure visuelle» que représente la classique note de cristallisation au cours du stockage.

D'autre part, nous avons étudié l'influence sur la note de 2 facteurs qui sont (ou devraient être) normalement maîtrisables en permanence au cours de la distribution à savoir :

- la température de stockage,

- la durée du stockage à température fixée, appelée «âge».

\section{MATÉRIELS ET MÉTHODES}

\section{Choix des miels et caractéristiques de départ}

Les 25 miels étudiés représentent pratiquement toutes les variétés de miels d'Europe occidentale (fig 5), sauf les miels de callune, qui sont très particuliers. Ce sont des miels du commerce, ayant tous subi l'analyse pollinique; 14 d'entre eux étaient plus ou moins cristallisés au départ. Ils ont été liquéfiés à $45^{\circ} \mathrm{C}$ avant analyse de leur composition en sucres par chromatographie en phase gazeuse (Pourtallier et Rognone, 1977). Leur viscosité a été mesurée aussitôt après, à chaque température de stockage, sur viscosimètre rotatif du type Couette à 30 vitesses de rotation. Tous les miels étudiés avaient un comportement newtonien. Leur teneur en eau a été mesurée par réfractométrie.

Ils ont ensuite été pasteurisés au laboratoire en tubes à essais de $70 \mathrm{~mm}$ de haut, maintenus sans agitation $45 \min$ à $78^{\circ} \mathrm{C}$.
Enfin, les tubes ont été stockés au repos dans des ambiances thermostatées à $5,10,15$ et $20^{\circ} \mathrm{C}$ (100 tubes au total).

\section{Mesures effectuées}

\section{Note de cristallisation}

Périodiquement, à 8 différents “âges" échelonnés de 25 jà 17 mois, chacun des 100 tubes a été observé visuellement et il lui a été attribué une note de cristallisation $N$ selon le barême de White et al (1962) rappelé dans le tableau I ( 800 notes ont été ainsi attribuées au total).

En même temps que $N$, la hauteur de la couche de cristaux $H(\mathrm{~mm})$ dans les tubes a également été estimée ( 800 valeurs de $H$ ).

\section{Sursaturation initiale, $\mathbf{S I}$}

Vers la fin de la période d'observation, pour un certain nombre de tubes, $N$ ou $H$ n'évoluaient plus. Il a été admis que ces tubes étaient parvenus à l'équilibre de saturation à la température de stockage où ils se trouvaient.

Pour 15 d'entre eux, nous avons réussi à séparer et doser les cristaux de glucose par polarisation, c'est-à-dire à donner une mesure de la sursaturation initiale en glucose (SI) du miel dans le tube. Deux expressions de SI ont été calculées :

(Glucose/Eau) du miel entièrement liquide initial

$\mathrm{SI}(\mathrm{Cl})=$

(Glucose/Eau) de la fraction liquide $\left(^{*}\right.$ ) à saturation

Glucose/poids du miel entièrement liquide initial (\%)

SI $(K)=\frac{}{\text { Glucose/poids de la fraction liquide }}$ à saturation $(\%)$

Ces valeurs sont approchées par défaut respectivement de celle de Claassen (Cl) et de Kucharenko (K) cités par Naveau (1953).

\footnotetext{
* On appelle généralement «liquidus» la fraction liquide.
} 
Tableau I. Degré de cristallisation (d'après White et al, 1962).

\begin{tabular}{llr}
\hline Note & Aspect & D/W \\
\hline & & \\
\hline 1 & Entièrement liquide & 1,58 \\
2 & Cuelques cristaux épars & 1,76 \\
3 & Quelques amas cristisée sur une hauteur de $1,5-3 \mathrm{~mm}$ & 1,79 \\
4 & Couche cristallisée sur une hauteur de $6-12 \mathrm{~mm}$ & 1,86 \\
5 & Échantillon cristallisé sur 1/4 de la hauteur du miel & 1,83 \\
6 & Échantillon cristallisé sur 1/2 hauteur du miel & 1,99 \\
7 & Échantillon cristallisé sur 3/4 hauteur du miel & 1,98 \\
8 & Échantillon entièrement cristallisé mou (aspect solide ou très visqueux) & 2,06 \\
& avec éventuellement une mince couche liquide surnageant (1-3 mm) & 2,16 \\
& Échantillon entièrement cristallisé dur & 2,24 \\
\hline
\end{tabular}

\section{L'activité de l'eau $a_{w}$}

Elle a été calculée pour chaque miel à partir de sa composition chimique par la méthode déjà éprouvée antérieurement (Tabouret, 1979; Tabouret et al, 1987).

\section{Les indices de composition chimique}

Ils sont tirés de l'analyse de la composition

$D=$ Glucose $/ \mathrm{miel}(\%)$

$L=$ Levulose $/$ miel $(\%)$

$W=$ Eau/miel $(\%)$

Indices : $D / W, I_{n}=\frac{D W}{\left(1-a_{w}\right)^{n}}$

Nous avons essayé d'optimiser $\mathrm{n}$ en lui attribuant les valeurs : $1,25 \quad 1,5 \quad 1,75 \quad 2$

L'indice L/D, utilisé par certains professionnels a aussi été calculé, mais il s'est révélé totalement redondant avec D/W ou In et il n'a pas été retenu dans l'étude statistique.

\section{Méthodes statistiques employées}

\section{Durée et température de stockage}

L'étude portant sur les 800 notes $N$ de cristallisation a été faite par analyse de la variance selon un modèle à 2 facteurs en randomisation totale :

$N_{i, j, k}=A+x_{i}^{D}+y_{j}^{T}+x y_{i, j}^{D, T}+z_{i, j, k}$

$N_{i, j, k}=$ valeur de la note de cristallisation;

$A=$ terme constant à toutes les notes;

$x_{i}^{D}=$ effet moyen de la durée de stockage $(i=$ 8);

$y_{i}^{T}=$ effet moyen de la température de stockage; $(j=4)$

$x y_{i, j}^{D, T}=$ effet moyen des intéractions durée $\mathrm{x}$ température de stockage;

$z_{i, j, k}=$ terme aléatoire dans le modèle;

$k=25$, nombre de répétitions.

Les comparaisons de moyennes ont été faites selon le test de Newmann-Keuls.

\section{Mesures instrumentales}

Leurs corrélations avec les notes $N$ à différents âges ont été calculées respectivement :

- à partir de 25 observations pour les descripteurs de la composition initiale (25 miels de composition initiale différente observés)

- à partir de 100 observations pour la viscosité initiale (25 miels $\times 4$ températures $=100$ viscosités initiales différentes observées).

L'examen des matrices de corrélation a permis de choisir les variables les plus performantes ainsi qu'une série de régressions linéaires multiples progressives pour expliquer au mieux la note $N$. 
Qu'il s'agisse de l'analyse de la variance ou des équations de régression de $N$, les coefficients de détermination $r^{2}$ donnent le pourcentage de variance expliqué par le modèle choisi $\left(r^{2}<100 \%\right)$.

Tous les calculs ont été effectués avec le logiciel STATITCF (1988).

\section{RÉSULTATS ET DISCUSSION *}

\section{Comparaison entre la note de crisallisation $\mathrm{N}$ et la hauteur de couche des cristaux $\mathrm{H}$}

Pour l'ensemble des 800 couples de valeurs $(N, H)$ observées, le coefficient de corrélation entre $N$ et $H$ est très hautement significatif $(r=0,979 P<0,001)$. Dans l'étude statistique ultérieure, il n'apparaît donc pas judicieux de considérer deux variables redondantes: seule la note $N$ sera conservée car c'est la variable usuelle en la matière, son emploi est plus commode et sa mesure s'avère de surcroît plus facile.

\section{Prévisibilité de la note de cristallisation N}

On peut dire que la prévisibilité pour l'intervalle de temps $T$ est bonne si les notes à 2 âges différant de $T$ sont bien correlées.

Nous avons étudié les différentes corrélations, tous miels et toutes températures confondus (25 miels $\times 4$ températures $=$ 100 observations) : la prévisibilité s'améliore quand $T$ diminue. Pratiquement, elle n'est acceptable qu'à court terme, pour $T$ ne dépassant guère 2 ou 3 mois, comme par exemple entre 3 et 6 mois ou entre 15 et 17 mois (fig 1) ce qui correspond respectivement à un stockage de moyenne durée et de longue durée.

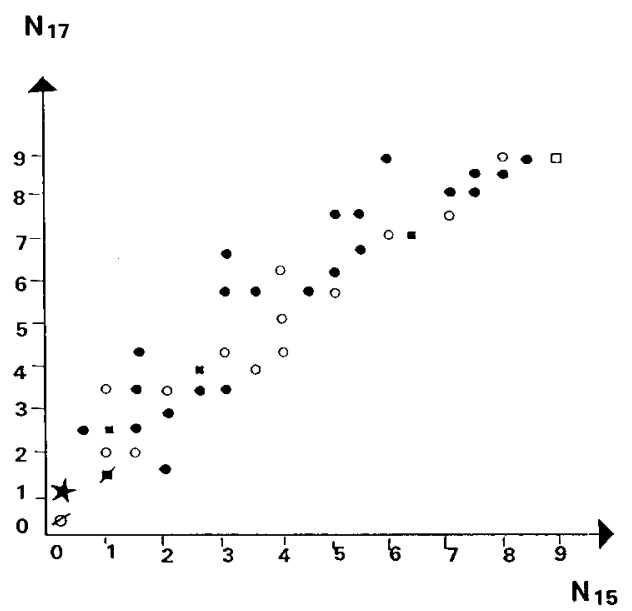

Fig 1. Comparaison entre les notes de cristallisation $N$ d'un même tube à 15 mois (abscisse) et à 17 mois (ordonnée). Nombre d'observations $n=100$ (100 tubes différents notés). $\star$ Ce point représente 23 tubes notés identiquement $(0$ à 15 mois puis 1 à 17 mois de stockage). $\sqcup 9$ tubes notés identiquement; 7 tubes notés identiquement; $\varnothing 4$ tubes notés identiquement; - 3 tubes notés identiquement; $O 2$ tubes notés identiquement; 1 tube noté.

La prévisibilité à long terme, par exemple entre 3 et 17 mois, ou, même à court terme mais après seulement $25 \mathrm{j}$ de stockage, n'est pas acceptable (fig 2).

Dans l'ensemble, la note actuelle ne permet donc pas de prévoir la note future, surtout au début du stockage.

\section{Influence de la durée et de la température de stockage sur la note de cristallisation $\mathrm{N}$}

L'analyse de la variance présentée dans le tableau II montre que la durée et la tempé-

\footnotetext{
* Nous tenons le fichier des résultats à la disposition des lecteurs qui le demandent.
} 


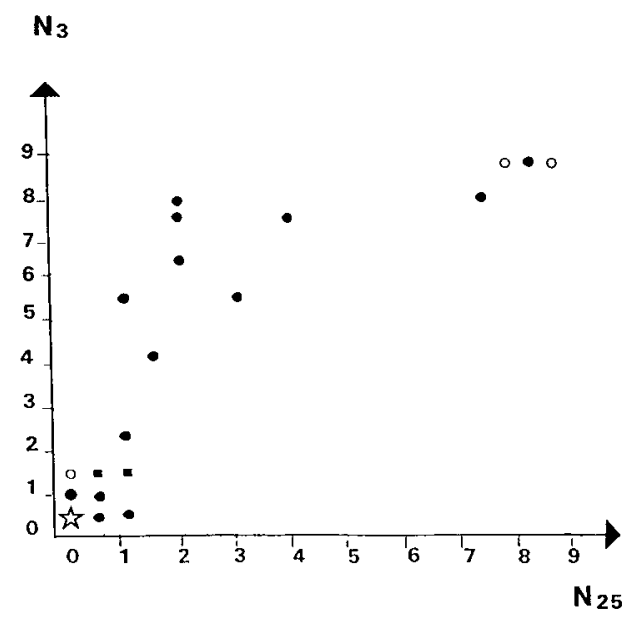

Fig 2. Comparaison entre les notes de cristallisation $N$ d'un même tube à 25 j (abscisse) et à 3 mois (ordonnée). Nombre d'observations $n=$ 100 (100 tubes différents notés). to point représente 74 tubes notés identiquement $(0$ à 25 j puis 0 à 3 mois de stockage). 3 tubes notés identiquement; $\mathrm{O} 2$ tubes notés identiquement; 1 tube noté.

rature de stockage influent de manière hautement significative $(P<0,001)$ sur $N$.

La comparaison des moyennes des notes, dans la colonne 5 du tableau II, montre qu'elles forment des groupes homogènes selon le test de Newman-Keuls, repérés par le même indice. En fonction de la température, 2 groupes seulement, $\mathrm{g}$ et $f$, sont significativement différents entre eux. En fonction de la durée, les moyennes des notes à 4,6 et 9 mois par exemple ne sont pas significativement différentes entre elles et forment le groupe $b$, mais la moyenne des notes à 3 mois (groupe a) est significativement différente de celle à 9 mois (groupes b et c).

Cependant, ces 2 facteurs ne contribuent qu'à l'explication d'une faible part de la variance de $N$ :
$-11,7 \%$ pour la durée;

$-3,3 \%$ pour la température.

Cela signifie que d'autres facteurs sont à considérer si l'on veut améliorer le coefficient de détermination du modèle qui est de $15 \%$ de la variance de la note de cristallisation.

Les influences sur $N$ des 2 facteurs sont présentées dans les figures 3 et 4 , où chacun des 32 points (4 températures $\times 8$ âges de miels) représente la valeur moyenne $N$ pour les 25 miels étudiés.

Malgré des écarts types moyens assez élevés (à 13 mois, $N=2,77 \pm 2,68$ ) on peut clairement voir sur la figure 3 la progression de la cristallisation avec le temps, pour l'ensemble des miels, à toutes les températures. Cette progression est à peu près linéaire. Elle est la plus rapide à $15^{\circ} \mathrm{C}$, mais sa vitesse (donnée par la pente de la courbe), ne change guère avec la température, même à $5^{\circ} \mathrm{C}$. Dans ce dernier cas, il y a simplement un «retard au démarrage" c'est-à-dire une période d'incubation plus longue, qui fait que $N$ est systématiquement plus basse. L'analyse de la variance de $N$ indique que les différences de $N$ en fonction des températures de stockage 10,15 ou $20^{\circ} \mathrm{C}$ ne sont pas significatives $(P<0,005)$. Seules des différences sont observées lors d'un stockage à $5^{\circ} \mathrm{C}$.

II paraît étonnant, au premier abord, que la vitesse de cristallisation soit pratiquement indépendante de la température. Cela peut néanmoins s'expliquer en analysant l'influence de la température sur 4 composantes essentielles des vitesses de nucléation et de croissance des cristaux (Tabouret, 1978), à savoir :

\section{Vitesse de migration}

des molécules dissoutes

Cette vitesse conditionne la disponibilité des molécules pour cristalliser. Elle est 
Tableau II. Analyse de la variance de la note de cristallisation du miel en fonction de la température et de la durée de stockage.

\begin{tabular}{|c|c|c|c|c|c|}
\hline & $\begin{array}{c}S C E \\
1\end{array}$ & $\begin{array}{l}D L \\
2\end{array}$ & $\begin{array}{l}F \\
3\end{array}$ & $r_{4}^{2}(\%)$ & 5 \\
\hline Variance totale & 6834 & 799 & & & \\
\hline $\begin{array}{l}\text { Variance «âge du miel» } \\
j=\text { jours } M=\text { mois }\end{array}$ & 799 & 7 & $15,14^{\star \star \star}$ & 11,7 & $\begin{array}{l}25 \mathrm{j}^{\mathrm{a}} 3 \mathrm{M}^{\mathrm{a}} 4 \mathrm{M}^{\mathrm{ab}} 6 \mathrm{M}^{\mathrm{ab}} 9 \mathrm{M}^{\mathrm{bc}} 13 \mathrm{M}^{\mathrm{cd}} \\
15 \mathrm{M}^{\mathrm{de}} 17 \mathrm{M}^{\mathrm{e}}\end{array}$ \\
\hline $\begin{array}{l}\text { Variance "température } \\
\text { de stockage" }{ }^{\circ} \mathrm{C}\end{array}$ & 224 & 3 & $9,9^{\star \star \star}$ & 3,3 & $10^{f} \quad 15^{f} \quad 20^{f}$ \\
\hline $\begin{array}{l}\text { Variance des interactions } \\
\text { "âge du miel" x } \\
\text { "température stockage" }\end{array}$ & 20 & 21 & $0,13 \mathrm{NS}$ & & \\
\hline Variance résiduelle & 5791 & 768 & & & \\
\hline
\end{tabular}

${ }^{1}$ Somme des carrés des écarts; ${ }^{2}$ degrés de liberté; ${ }^{3} F$ de Fisher et seuil de signification; ${ }^{* *} P<0,001$ et NS $=$ non significatif; ${ }^{4}$ coefficient de détermination; ${ }^{5}$ comparaison des moyennes des notes $(25 \mathrm{j}=$ moyenne à 25 jours, $3 \mathrm{M}=$ moyenne à 3 mois, etc.) : les niveaux de même indice (b par exemple) forment des groupes homogènes selon le test de Newmann-Keuls. Ces groupes peuvent partiellement se recouper (indices ab, bc... par exemple).

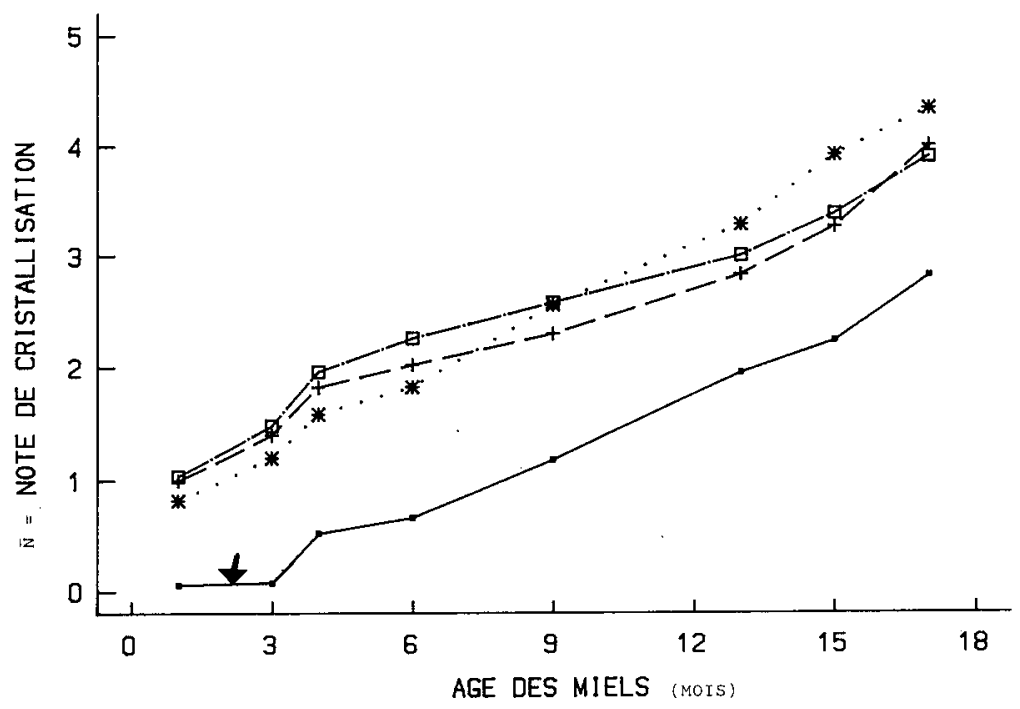

Fig 3. Évolution de la note en fonction de la durée du stockage à température fixée. $N$ est la moyenne des 25 miels étudiés. $-\longrightarrow$ Stockage à $5^{\circ} \mathrm{C}$ - $\longrightarrow$ - Stockage à $10^{\circ} \mathrm{C} \ldots$. . . . . Stockage à $15^{\circ} \mathrm{C}$. - . - - Stockage à $20^{\circ} \mathrm{C}$. La flèche indique le retard au démarrage de la cristallisation. 


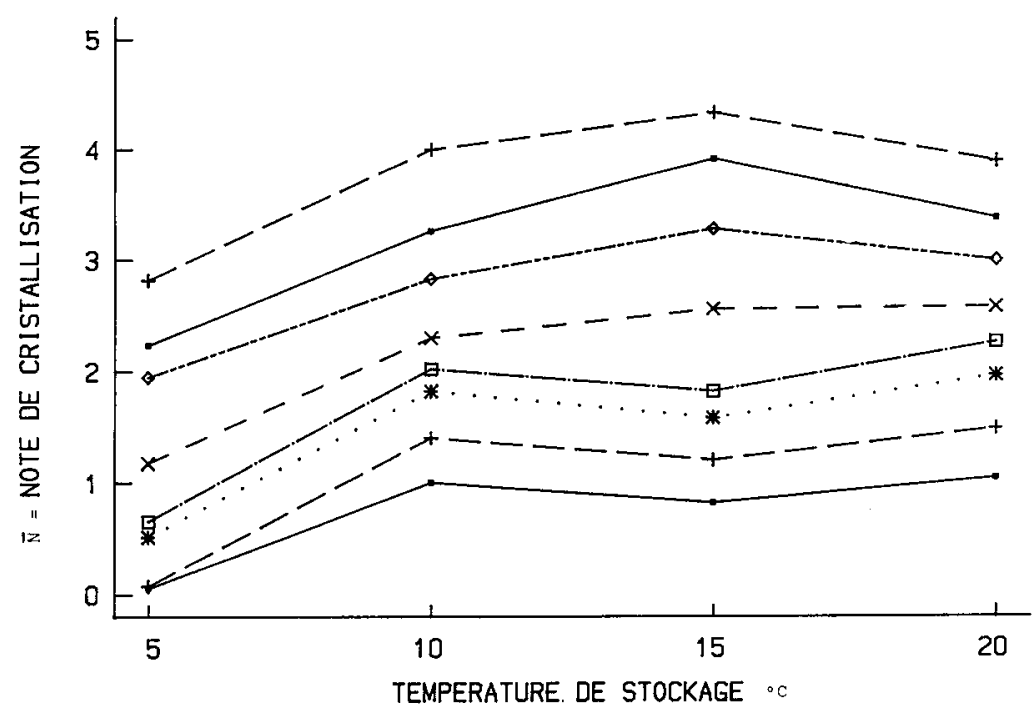

Fig 4. Variation de la note de cristallisation $\mathrm{N}$ avec la température du stockage, pour une durée de stockage fixée. $N$ est la moyenne des 25 miels étudiés._- - stockage $25 \mathrm{j}$; - - stockage 3 mois;

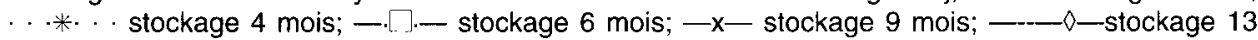
mois; —- stockage 15 mois; -_+- stockage 17 mois.

elle-même conditionnée par la viscosité du liquidus.

\section{Vitesse de mutarotation} glucose $\beta$--> glucose $\alpha$

Seul le glucose $\alpha$ peut cristalliser (sous forme $\alpha$-hydrate).

\section{Vitesse de pénétration et d'insertion} des molécules de glucose $\alpha$ dans les germes ou cristaux déjà formés

\section{Sursaturation du liquidus en glucose $\alpha$}

À basse température, les 3 vitesses de migration, de mutarotation et d'insertion sont réduites, ce qui freine le processus de cristallisation, mais la sursaturation est élevée, ce qui est très favorable à la cristallisation.
À température plus élevée, les 3 vitesses mentionnées sont plus grandes, mais la sursaturation est plus faible. II apparaît donc que, dans nos conditions d'expérience, et notamment en l'absence d'agitation et d'ensemencement, les tendances opposées se compensent sensiblement.

La période d'incubation constatée à $5{ }^{\circ} \mathrm{C}$ est à attribuer, à notre avis, à l'influence particulière de la viscosité qui serait prépondérante au début du processus de cristallisation et en constituerait pendant un certain temps le facteur limitant : voir l'analyse approfondie du rôle de la viscosité dans l'évolution à température constante proposée cidessous. 
Relations entre la note de cristallisation $\mathrm{N}$ et les mesures instrumentales (composition initiale et viscosité initiale des miels)

\section{Corrélation entre la note de cristallisation $N$ et la viscosité initiale des miels}

On voit dans le tableau III que ces corrélations faibles sont pourtant significatives $(P<0,01)$, ce qui laisse supposer l'existence d'un lien entre la viscosité initiale et $N$.

Nous pensons même que ce lien pourrait être plus important qu'il n'y paraît ici, pour les raisons suivantes:

- la viscosité est fortement corrélée à la température; elle varie en sens inverse : pour les 100 observations : $r=-0,745 ; P<$ 0,001 ;

- or, à basse température $\left(5^{\circ} \mathrm{C}\right)$, cette viscosité initiale est élevée et pourrait constituer alors un facteur limitant de la cristallisation. Cette hypothèse explique alors pourquoi les différents tubes à $5{ }^{\circ} \mathrm{C}$ restent tous liquides au début du stockage : ils ne peuvent se différencier en fonction de la viscosité que progressivement, dans la mesure justement où la cristallisation peut se développer. II faut rappeler dans le même ordre d'idées que les miels liquides quels qu'ils soient, s'ils sont congelés et conservés à $-15^{\circ} \mathrm{C}$, ne cristallisent pas : leur viscosité excessive constitue un facteur limitant permanent.

Ainsi, au début du processus, le rôle de la viscosité peut très bien être plus ou moins masqué.

D'autre part, en considérant dans ce qui suit un processus d'évolution au cours du stockage à température constante :

- au début du stockage, la viscosité du liquidus est maximale. Elle ne peut en effet que diminuer s'il y a cristallisation, car alors le liquidus s'appauvrit en matière sèche : par exemple, une estimation, basée sur nos travaux antérieurs (Tabouret, 1980), montre que la viscosité du liquidus $\mathrm{n}^{\circ} 43$ (miel de tournesol stocké à $15^{\circ} \mathrm{C}$ ) est ainsi divisée par 2,4 environ pendant la cristallisation. Cette diminution de viscosité, comme on l'a vu, est favorable à la migration des molécules de glucose dissoutes et donc à la cristallisation;

- mais par la suite, d'autres facteurs interviennent simultanément et de plus en plus sur les différences d'évolution entre les tubes:

- la quantité de cristaux déjà formés dans un tube augmente la vitesse de cristallisation dans ce tube, car celle-ci est proportionnelle à la surface des cristaux. De ce fait, la cristallisation tend à s'accélérer d'elle-même (c'est peut-être une explication de l'accélération qu'on peut constater sur la figure 3 entre 3 et 4 mois);

Tableau III. Coefficients de corrélation r entre la viscosité initiale des miels et $N$, toutes températures confondues; $n=100$ observations.

\begin{tabular}{lllllllll}
\hline Durée de stockage * $25 \mathrm{~J}$ & $3 M$ & $4 M$ & $6 M$ & $9 M$ & $13 M$ & $15 M$ & $17 M$ \\
\hline$r$ & $-0,19$ & $-0,21$ & $-0,21$ & $-0,22$ & $-0,24$ & $-0,23$ & $-0,23$ & $-0,24$
\end{tabular}

* $J=$ jour; $M=$ mois 
- mais en même temps, la diminution de la sursaturation du liquidus ralentit la cristallisation;

- finalement, on constate sur les figures 3 et 4 que, malgré leur intensité croissante, les effets opposés se compensent et donnent grosso modo une vitesse de cristallisation pratiquement constante.

On voit donc que l'influence éventuelle de la viscosité initiale peut se trouver occultée aussi quand le stockage isotherme se prolonge, par la progression d'autres influences complexes.

En conclusion, il est plausible que la viscosité initiale des miels soit un facteur limitant temporaire de cristallisation à la température de $5{ }^{\circ} \mathrm{C}$, mais non aux températures plus élevées, ce qui peut expliquer le «retard d'incubation" précédemment constaté sur tous les tubes stockés à $5{ }^{\circ} \mathrm{C}$ et l'évolution, pratiquement indépendante de la température, de tous les tubes après incubation.

\section{Corrélations entre la note de cristallisation $N$ et la composition initiale des miels}

Dans le tableau IV (réparti en 4 soustableaux) des corrélations à chaque température de stockage, on remarque tout d'abord que la teneur en eau $W$ et l'activité de l'eau $a_{w}$ n'ont pas de corrélation significative avec $N$ puisque $r$ est constamment inférieur au seuil de $0,396(P<0,05)$. Cela est dû à la faible variabilité de ces facteurs dans les miels testés (Tabouret et al, 1987, p 17). II en résulte aussi qu'il y a peu de différence entre les corrélations avec $\mathrm{N}$ des autres descripteurs de la composition initiale que sont $D, D / W, L / D$ et $I_{n}(n=$ 1,25-2). Nous choisirons donc comme descripteur unique l'indice $I_{1,25^{\prime}}$ parce qu'il s'est révélé plus performant pour expliquer $N$ quand la teneur en eau est plus variable (Tabouret, 1979, Tabouret et al, 1987).

\section{Essais de régression de la note de cristallisation $N$ sur les mesures instrumentales}

En plus de l'indice $I_{1,25}$ précédemment choisi, il est intéressant de retenir comme deuxième variable explicative de $N$ la viscosité initiale des miels. Son influence plausible a déjà été évoquée; et, de plus, elle est statistiquement indépendante de $I_{1,25}$ (pour 100 observations, $r=0,008$; non significatif).

On peut voir, dans l'exemple fourni par le tableau $V$ des notes à 15 mois que cette introduction de la viscosité peut être justifiée pour les températures de stockage de 10,15 et $20^{\circ} \mathrm{C}$, puisque le coefficient de détermination $r^{2}$, qui devient $r^{2} m$, s'accroît de ce fait de $13 \%$ à $15{ }^{\circ} \mathrm{C}$ et $20^{\circ} \mathrm{C}$, car il passe respectivement de 0,452 à 0,511 et de 0,405 à 0,456 , et de $17 \%$ à $10^{\circ} \mathrm{C}$ (il passe de 0,312 à 0,365 ).

La comparaison entre la variance expliquée dans le modèle faisant intervenir la température plus la durée de stockage $(15,0 \%)$ et celle expliquée dans le modèle utilisant l'indice I plus la viscosité $\left(r_{m}^{2} \mathrm{com}\right.$ pris entre 0,256 et 0,511 ) montre le rôle important de la composition et celui plus limite de la température et de la durée de stockage.

Les régressions présentées dans ce tableau $\mathrm{V}$ permettent d'estimer quelle sera la note à 15 mois d'âge, à partir du moment où l'on a mesuré la composition et la viscosité du miel liquide avant tout traitement et avant tout stockage. À titre d'exemple, la note à prévoir après un stockage de 15 mois à $15^{\circ} \mathrm{C}$ se calcule par la formule $F^{2}$

$$
\begin{aligned}
F^{2} X & =(2,903 \pm 0,691) \times l_{1,25} \\
& -(0,032 \pm 0,020) \times \text { viscosité }-12,618
\end{aligned}
$$

Sur la figure 5, pour chacun des 25 miels, la note individuelle réellement obtenue est 
Tableau IV. Corrélations entre la note de cristallisation $N$, à différentes températures de stockage des 25 miels, et les analyses instrumentales. Les seuils de signification des coefficients de corrélation sont de : 0,$396 ; P<0,05$ et $0,505, P<0,01$ pour $n=25$ miels.

\begin{tabular}{lrrrrrrrrrr}
\hline$T_{1}=5^{\circ} C$ & VISCO & $W$ & $a_{W}$ & $L$ & $D$ & $D / W$ & $l_{t, 25}$ & $I_{1,50}$ & $I_{1,75}$ & $I_{2,00}$ \\
$N_{25}$ & $-0,107$ & 0,130 & $-0,162$ & 0,177 & 0,536 & 0,522 & 0,526 & 0,521 & 0,512 & 0,501 \\
$N_{3}$ & $-0,029$ & 0,056 & $-0,149$ & 0,157 & 0,352 & 0,357 & 0,344 & 0,337 & 0,328 & 0,317 \\
$N_{4}$ & $-0,096$ & 0,211 & $-0,058$ & 0,099 & 0,543 & 0,512 & 0,552 & 0,554 & 0,554 & 0,551 \\
$N_{6}$ & $-0,085$ & 0,187 & $-0,117$ & 0,139 & 0,593 & 0,567 & 0,592 & 0,590 & 0,586 & 0,579 \\
$N_{9}$ & $-0,135$ & 0,206 & $-0,084$ & 0,119 & 0,574 & 0,542 & 0,579 & 0,581 & 0,580 & 0,576 \\
$N_{13}$ & $-0,178$ & 0,261 & 0,004 & 0,073 & 0,545 & 0,493 & 0,555 & 0,562 & 0,567 & 0,569 \\
$N_{15}$ & $-0,181$ & 0,264 & 0,049 & 0,027 & 0,482 & 0,426 & 0,496 & 0,506 & 0,514 & 0,520 \\
$N_{17}$ & $-0,197$ & 0,292 & 0,096 & 0,042 & 0,439 & 0,371 & 0,451 & 0,464 & 0,475 & 0,485
\end{tabular}

$T_{2}=10^{\circ} \mathrm{C}$

$\begin{array}{lllllllllll}\mathrm{N}_{25} & -0,262 & 0,050 & -0,050 & 0,044 & 0,196 & 0,192 & 0,201 & 0,200 & 0,198 & 0,195 \\ \mathrm{~N}_{3} & -0,233 & 0,024 & -0,142 & 0,108 & 0,294 & 0,307 & 0,294 & 0,288 & 0,279 & 0,269 \\ \mathrm{~N}_{4} & -0,254 & 0,044 & -0,150 & 0,118 & 0,351 & 0,361 & 0,352 & 0,345 & 0,336 & 0,326 \\ \mathrm{~N}_{6} & -0,271 & 0,035 & -0,176 & 0,115 & 0,388 & 0,405 & 0,390 & 0,381 & 0,371 & 0,358 \\ \mathrm{~N}_{9} & -0,298 & 0,067 & -0,163 & 0,113 & 0,433 & 0,443 & 0,437 & 0,430 & 0,421 & 0,409 \\ \mathrm{~N}_{13} & -0,306 & 0,067 & -0,189 & 0,119 & 0,487 & 0,501 & 0,491 & 0,483 & 0,472 & 0,458 \\ \mathrm{~N}_{15} & -0,319 & 0,099 & -0,188 & 0,126 & 0,554 & 0,561 & 0,559 & 0,551 & 0,541 & 0,528 \\ \mathrm{~N}_{17} & -0,289 & 0,046 & -0,235 & 0,089 & 0,551 & 0,575 & 0,559 & 0,548 & 0,535 & 0,518\end{array}$

$T_{3}=15^{\circ} \mathrm{C}$

$\begin{array}{lllllllllll}\mathrm{N}_{25} & -0,185 & 0,080 & -0,050 & 0,077 & 0,245 & 0,233 & 0,246 & 0,245 & 0,243 & 0,240 \\ \mathrm{~N}_{3} & -0,129 & 0,072 & -0,150 & 0,159 & 0,391 & 0,392 & 0,386 & 0,380 & 0,371 & 0,361 \\ \mathrm{~N}_{4} & -0,170 & 0,107 & -0,124 & 0,148 & 0,421 & 0,414 & 0,420 & 0,416 & 0,410 & 0,401 \\ \mathrm{~N}_{6} & -0,190 & 0,149 & -0,111 & 0,165 & 0,478 & 0,462 & 0,477 & 0,475 & 0,470 & 0,463 \\ \mathrm{~N}_{9} & -0,277 & 0,236 & -0,047 & 0,153 & 0,550 & 0,510 & 0,554 & 0,558 & 0,558 & 0,557 \\ \mathrm{~N}_{13} & -0,321 & 0,299 & -0,032 & 0,137 & 0,669 & 0,615 & 0,679 & 0,686 & 0,689 & 0,689 \\ \mathrm{~N}_{15} & -0,345 & 0,291 & -0,049 & 0,174 & 0,664 & 0,614 & 0,672 & 0,677 & 0,679 & 0,677 \\ \mathrm{~N}_{17} & -0,283 & 0,214 & -0,110 & 0,120 & 0,648 & 0,623 & 0,661 & 0,662 & 0,659 & 0,653\end{array}$

\begin{tabular}{lllllllllll}
$T_{4}=20^{\circ} \mathrm{C}$ & \multicolumn{1}{c}{$\boldsymbol{\downarrow}$} \\
$\mathrm{N}_{25}$ & $-0,141$ & 0,065 & $-0,103$ & 0,112 & 0,303 & 0,301 & 0,302 & 0,298 & 0,293 & 0,286 \\
$N_{3}$ & $-0,110$ & 0,028 & $-0,158$ & 0,139 & 0,316 & 0,329 & 0,313 & 0,306 & 0,296 & 0,285 \\
$N_{4}$ & $-0,176$ & 0,092 & $-0,150$ & 0,153 & 0,440 & 0,439 & 0,439 & 0,433 & 0,425 & 0,415 \\
$N_{6}$ & $-0,205$ & 0,094 & $-0,178$ & 0,155 & 0,501 & 0,504 & 0,501 & 0,494 & 0,485 & 0,472 \\
$N_{9}$ & $-0,253$ & 0,133 & $-0,151$ & 0,148 & 0,538 & 0,531 & 0,541 & 0,536 & 0,529 & 0,519 \\
$N_{13}$ & $-0,300$ & 0,158 & $-0,147$ & 0,157 & 0,580 & 0,568 & 0,585 & 0,581 & 0,575 & 0,565 \\
$N_{15}$ & $-0,328$ & 0,172 & $-0,163$ & 0,179 & 0,631 & 0,618 & 0,636 & 0,632 & 0,624 & 0,614 \\
$N_{17}$ & $-0,349$ & 0,185 & $-0,141$ & 0,166 & 0,618 & 0,600 & 0,625 & 0,623 & 0,618 & 0,610
\end{tabular}

Le descripteur choisi pour représenter la composition initiale des miels est I1,25, la colonne correspondante est repérée par la flèche VISCQ : viscosité des miels; $W$ : teneur en eau des miels; $a_{w}=$ activité de l'eau des miels; $L:$ teneur en en lévulose des miels; $D$ : teneur en glucose des miels; $I_{n}=1,25-2$ : indices de composition (voir $2-7$ ); $N_{25}$ note après 25 j de stockage; $N_{m=3-17}$ : note après m mois de stockage. 
Tableau V. Coefficients des équations de régression calculées entre la note $N$ à 15 mois d'une part et $l_{1,25}$ et la viscosité des miels d'autre part.

\begin{tabular}{ccccc}
\hline & $5{ }^{\circ} \mathrm{C}$ & $10{ }^{\circ} \mathrm{C}$ & $15{ }^{\circ} \mathrm{C}$ & $20{ }^{\circ} \mathrm{C}$ \\
\hline a & $2,120 \pm 0,825$ & $2,924 \pm 0,969$ & $2,903 \pm 0,691$ & $3,160 \pm 0,842$ \\
b & $-0,002 \pm 0,004$ & $-0,015 \pm 0,011$ & $-0,032 \pm 0,020$ & $-0,074 \pm 0,051$ \\
c & $-10,148$ & $-13,234$ & $-12,618$ & $-14,597$ \\
$r$ & 0,496 & 0,559 & 0,672 & 0,636 \\
$r^{2}$ & 0,246 & 0,312 & 0,452 & 0,405 \\
$r_{m}$ & 0,509 & 0,604 & 0,715 & 0,675 \\
$r^{2}{ }_{m}$ & 0,256 & 0,365 & 0,511 & 0,456 \\
$p$ & 52,8 & 19,1 & 11,2 & 15,7 \\
\hline
\end{tabular}

Note $=a I_{1,25}+b$ viscosité $+c$

$r^{2}=$ coefficient de détermination simple (calculé avec I1,25 seulement); $r_{m}, r_{m}^{2}=$ coefficient de corrélation multiple, coefficient de détermination multiple (calculé avec 11,25 et viscosité); $p=$ probabilité (\%) à l'introduction non justifiée de la seconde variable (viscosité) dans l'équation de régression.

figurée en regard de la note $X$ calculée par l'équation de régression $F^{2}$.

Dans le tableau $V$, on voit aussi que l'indice de composition chimique $I_{1,25}$, auquel correspond le coefficient de détermination simple $r^{2}$, explique jusqu'à $45 \%$ de la variabilité de $N$. Ce résultat concorde tout à fait avec ceux trouvés antérieurement (Tabouret et al, 1987).

En tout état de cause, cependant, on constante que malgré l'introduction de la viscosité, le coefficient de détermination multiple plafonne, dans les meilleurs cas, autour de $r_{m}{ }^{2}=50 \%$, ce qui signifie que $50 \%$ de la variabilité de $N$ restent inexpliqués.

Même dans le cas particulier des 15 tubes dont nous avons mesuré la sursaturation initiale $S I$, nous avons constaté que l'introduction de cette variable supplémentaire $S I$ dans l'équation de régression (à côté de $I_{n}$ et de la viscosité) n'est pas justifiée statistiquement et ne peut améliorer le coefficient de détermination.
D'autre part, en étudiant attentivement la figure 5 , on s'aperçoit que seuls quelques miels ont un comportement atypique: soit ceux qui ont cristallisé très rapidement («Jura» et "Gâtinais»: notes très rapidement voisines de 9); soit ceux qui n'ont jamais cristallisé («Acacia», "toutes fleurs" et «202»: notes de 0 ou 1 à 15 mois). Si l'on élimine ces miels dans la régression multiple de l'exemple (stockage à $15^{\circ} \mathrm{C}$ ), le coefficient de régression associé $r_{m}^{2}$ passe de 0,511 à 0,788 . Cette constatation nous a incités à envisager une étude statistique plus détaillée, dans laquelle quelques familles de miels seront étudiées à part.

\section{CONCLUSION}

Dans ce nouvel essai d'approche statistique des facteurs de la tendance des miels à cristalliser, nous avons étendu le champ d'expérimentation en diversifiant les miels 


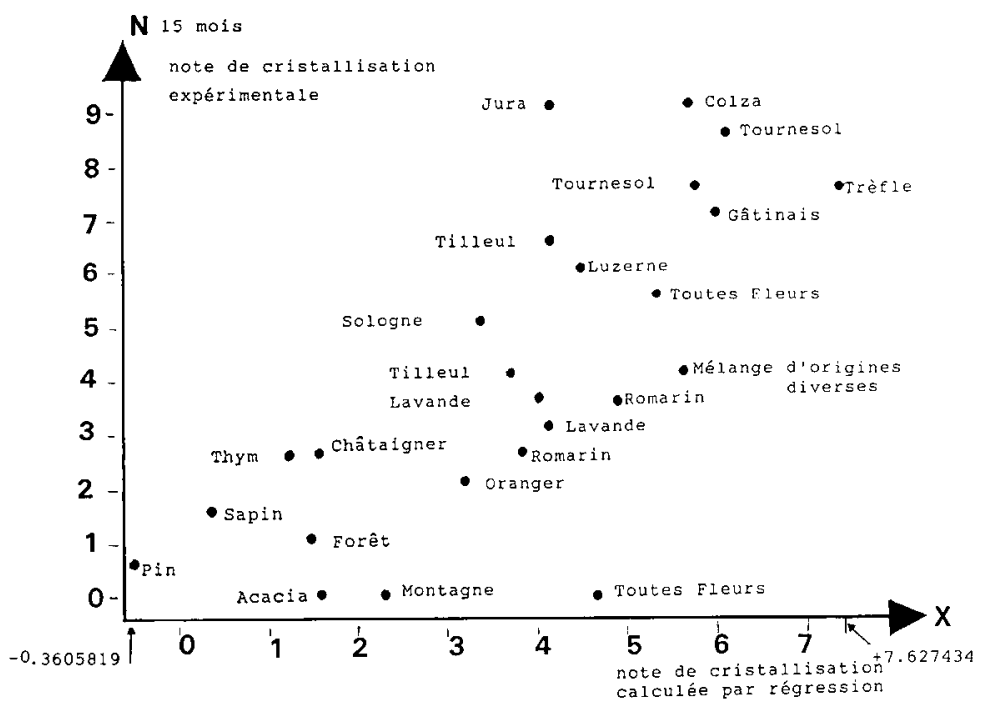

Fig 5. Comparaison entre notes prédites avant stockage et notes réelles pour un stockage de 15 mois à $15^{\circ} \mathrm{C} . X=$ note calculée par la formule de régression multiple. minimum $-0,3605819$; maximum $=+7,627434 ; N_{15}=$ note réelle; minimum 0 ; maximum 9; nombre d'observations : 25 (25 miels); nombre de variables : $4\left(l_{1,25}\right.$ représentant la composition initiale, viscosité initiale, $\left.X, N_{15}\right)$.

étudiés, en recherchant les influences possibles de la viscosité initiale des miels, de la température de stockage et de sa durée.

L'étude a montré la médiocre prévisibilité, en général, de l'évolution de la cristallisation à partir de la note actuelle de cristallisation, sauf à court terme, et même à court terme si le miel est de fabrication récente.

D'autre part, un résultat aussi intéressant qu'inattendu, est que, après incubation, la vitesse de cristallisation (représentée par l'accroissement de la note au cours du temps) ne dépend pratiquement pas de la température de stockage entre $5{ }^{\circ} \mathrm{C}$ et $20^{\circ} \mathrm{C}$. L'apiculteur peut en tirer des applications concrètes intéressantes : s'agissant de commercialiser un miel liquide qui a commencé à grainer, il ne peut espérer ralentir la progression de la cristallisation en le transférant en chambre froide si les autres facteurs $\left(I_{1,25}\right.$ et éventuellement viscosité) laissent prévoir des notes élevées. S'agissant au contraire de préparer un miel crémeux, qui est donc ensemencé, le séjour préconisé empiriquement par Dyce (1931) en chambre froide se justifie pleinement, malgré son surcoût, car il assure une "prise en masse" tout aussi rapide qu'à $14^{\circ} \mathrm{C}$, mais avec des cristaux plus fins grâce à la sursaturation très élevée, qui confère au produit final une meilleure qualité gustative.

Globalement, malgré tous les facteurs connus pris en compte dans l'étude, la va- 
riabilité de la note de cristallisation n'est expliquée, dans les meilleurs cas, qu'à hauteur de $50 \%$ par ces facteurs. Certains types de miel cristallisent très facilement en toutes circonstances (exemple "colza»); d'autres ne cristallisent pour ainsi dire jamais, quelles que soient les circonstances (ex : acacia). Ces comportements atypiques sont partiellement responsables des indéterminations rencontrées, puisque le coefficient de détermination $r^{2}$ s'élève à près de $80 \%$ si on écarte de l'étude ces quelques miels atypiques qui doivent désormais être étudiés à part. Une analyse plus fine de la composition des miels permettrait vraisemblablement de mieux comprendre le déterminisme de leur cristallisation.

Dans les cas les plus favorables, (le stockage de 15 mois est traité en exemple), les régressions établies sur la composition en sucres et eau et sur la viscosité initiales permettent, dès le jour de la réception du miel, d'estimer grossièrement la note qu'il est susceptible d'obtenir $6,9,13$ ou 15 mois après sa mise en pots si les pots sont stockés immobiles et à température constante. Cette note peut être regardée en somme comme un potentiel de cristallisation propre au miel considéré, indépendamment des traitements qu'on lui applique. Sa prise en compte pourrait donc s'avérer utile aussi dans l'assemblage initial des lots disponibles pour composer le miel qui, une fois pasteurisé et conditionné avec soin, aura le moins de chance de cristalliser au cours de la distribution.

\section{Summary - Tendency of honeys to crystallize: a renewed attempt at a sta- tistical approach. This study, which con- tinues the 1987 investigation, has attempt- ed to correlate the tendency of market honeys to crystallize with technological production data that can be measured but not always controlled. Twenty-five typical}

honeys from Western Europe were liquified, heated at $78{ }^{\circ} \mathrm{C}$ for pasteurization and placed in 100 glass tubes which were kept at $5,10,15$ and $20^{\circ} \mathrm{C}$. Each tube was observed at 8 different times for 17 months, and was graded from 0 (none) to 9 (hard crystallized) ( 800 gradings in all). The statistics cover five tables, with an analysis of variance of the grade together with the storage temperature and duration (table II), and multiple stepwise linear regressions of the grade on the initial composition and viscosity of liquid honey (table III), as it stands just before pasteurization.

The development of graining is shown in figures 3 and 4 . There is no significant difference between storage temperatures of 10,15 or $20^{\circ} \mathrm{C}$. Storage at $5^{\circ} \mathrm{C}$ begins with a so-called "latent" time without any crystallization, but then graining will develop at the same constant rate as the other temperatures. These results are discussed in terms of crystallization technology; an important but not obvious role of the initial viscosity is presumed.

At the beginning of storage, the grade cannot be predicted from the controlled factors. In case of longer storage times, on the contrary, the grade may be predicted from the initial composition and viscosity of the honey. As an example, a regression formula is given for 15 months storage at $15^{\circ} \mathrm{C}$, wherein the calculated grades are in rather good agreement with the observed true grades (fig 5). The grade variability is also rather well explained - about $80 \%$ - when considering the majority of honeys, but a few varieties present atypical behaviour: whatever the storage temperature and time, rape honeys always crystallized very fast, whereas Robinia honeys never crystallized. This is why, when considering the entire batch of honeys, only $50 \%$ of the variability is explained. Improvement of this score would undoubtedly require a better knowledge of the factors which play a part in graining tendency, especially more thor- 
ough compositional analyses of a wide variety of honeys.

\section{honey / crystallization / storage / statis- tics}

\section{Zusammenfassung - Die kristallisie-} rungstendenz der Honige: neuer Versuch einer statistischen Annäherung. In dieser Studie, die eine Untersuchung von 1987 fortsetzt, wird der Versuch unternommen, die Kristallisierungsstendenz von Markthonigen mit Produktions-daten $\mathrm{zu}$ korrelieren, die gemessen werden können, obwohl sie nicht immer kontrollierbar sind. 25 typische Honige aus Westeuropa wurden verflüssigt, zur Pasteurisation mit $78^{\circ} \mathrm{C}$ überhitzt und schließlich in 100 Glasröhrchen abgefüllt und bei Temperaturen von $5,10,15$ und $20^{\circ} \mathrm{C}$ aufbewahrt. Jedes Röhrchen wurde achtmal zu verschiedenen Zeiten inspiziert, bis zu 17 Monate lang; bei jeder Inspektion wurden die Röhrchen in einer Skala von 0-9 klassifiziert (insgesamt 800 Zensuren). Die statistische Analyse umfaßte in fünf Tabellen eine Varianzanalyse der Klassifizierung mit der Aufbewahrungstemperatur und der Dauer (Tabelle II) und eine multiple schrittweise lineare Regression der Klassen über die Daten der ursprünglichen Zusammensetzung und Viskosität des flüssigen Honigs unmittelbar vor der Pasteurisierung (Tabelle III).

Die Entwicklung des Körnigwerdens des Honigs ist in Abbildungen 3 und 4 dargestellt. Es besteht kein signifikanter Unterschied zwischen den Lagerungstemperaturen von 10,15 oder $20^{\circ} \mathrm{C}$. Die bei $5^{\circ} \mathrm{C}$ aufbewahrten Proben verhalten sich anders: sie beginnen mit einer sogenannten 'Latenzperiode' ohne jede Kristallisierung, aber nach dieser Zeit entwickelt sich die Körnerbildung ganz mit derselben konstanten Rate wie bei den anderen Temperaturen. Diese Resultate werden in Bezie- hung zur Kristallisationstechnologie diskutiert. Es kann eine wichtige, obwohl unauffällige Rolle der ursprünglichen Viskosität angenommen werden.

Bei Beginn der Lagerung kann der spätere Klassenwert aus den kontrollierten Faktoren nicht vorausgesagt werden. Bei längeren Lagerungszeiten lässt sich der Wert jedoch aus der ursprünglichen Zusammensetzung und der Viskosität des Honigs voraussagen. Als Beispiel wird eine Regressionsformel für eine Lagerung über 15 Monate bei $15^{\circ} \mathrm{C}$ gegeben, wobel die errechneten Werte gut mit den tatsächlich beobachteten übereinstimmen (Abbildung 5). Bei der Mehrzahl der Honige läßt sich die Variabilität der Werte gut - zu $80 \%$ - erklären; aber einige Honige zeigen ein abweichendes Kristallisationsverhalten: Rapshonige kristallisierten immer sehr schnell, bei welcher Lagerungstemperatur und Zeit auch immer, während Akazien(Robinien-)Honige niemals kristallisierten. Deshalb kann bei Betrachtung der Gesamtheit der Honige nur 50\% der Variabilität erklärt werden. Zu einer Verbesserung dieses Ergebnisses wären ohne Zweifel erweiterte Kenntnisse über die an der körnerbildung beteiligten Faktoren notwendig; vor allem wären noch viel genauere Analysen über die Zusammensetzung erforderlich, um der außerordentlich komplizierten Variabilität der Honige gewachsen zu sein.

\section{Honig / Kristallisierung / Lagerung / Sta- tistik}

\section{RÉFÉRENCES}

Dyce EJ (1931) Fermentation and crystallization of honey. Bull Cornell Univ Agric Exp Stn $n^{\circ}$ $528,52-75$

Gonnet M (1965) Les modifications de la composition chimique des miels au cours de la conservation. Ann Abeille 8, 129-146 
Naveau J (1953) Les diverses définitions de la sursaturation. In: Com Int Tech Sucrerie. Assemblée Générale 71953 Bruxelles. La cristallisation du sucre. Tienen CITS secrétariat Général, 39-40

Pourtallier J, Rognone C (1977) Méthode modifiée de dosage des sucres des miels par chromatographie en phase gazeuse. Symp Int Technol Apic Bologne. 25-27 janvier 1977. Apimondia Bucarest, 73-83

Statitcf 88 Logiciel d'analyse des données Inst technique des céréales et des fourrages -8 avenue du Président Wilson, 75116 Paris

Tabouret T (1978) Technologie de la cristallisation. Aspects théoriques et pratiques dans quelques industries alimentaires. CDIUPA 91305 Massy 17, 51-53 (116 pages)

Tabouret T (1979) Rôle de l'activité de l'eau dans la cristallisation du miel. Apidologie 10, 341-358

Tabouret (1980) Contribution à l'étude fondamentale de la pasteurisation du miel et des solutions aqueuses sursaturées de $D$ glucose. Thèse Doct Sci, Univ Dijon $110 p$

Tabouret T, Carteron A, Lheritier J (1987) La tendance des miels à cristalliser : un essai d'approche statistique. Apidologie 18, 11-26

White J, Riethof M, Subers M, Kushnir I (1962) Composition of American honeys. Tech Bull US Dept Agric n $1261,124 \mathrm{p}$ 